\title{
mAbES: Avaliação de uso de uma aplicação móvel para usuários que são cegos
}

\author{
Marcia de Borba Campos, Juliana Damásio, Tasmay Inácio \\ Faculdade de Informática - Pontifícia Universidade Católica do Rio Grande do Sul \\ (PUCRS) - Porto Alegre - RS - Brasil \\ marcia.campos@pucrs.br, \{juliana.damasio, tasmay.inacio\}@acad.pucrs.br
}

\begin{abstract}
In this paper, we discuss results of evaluations of a mobile gaming application (mAbES) for user who are blind. The mAbES was evaluated with the participation of HCI specialists and experts in videogaming (Study 1) and $m A b E S$ end-users who are blind (Study 2). The instruments used by the Study 1 were the audio feedback questionnaire and the usability evaluation questionnaire. It also includes questions based on video games and gaming mechanics. For Study 2 the audio evaluation questionnaire and the O\&M, tactile feedback, easiness of use questionnaire were applied. Semi-structured interviews were also applied.
\end{abstract}

Resumo. Este artigo apresenta a avaliação de usabilidade de uma aplicação mobile (mAbES) para pessoas com deficiência visual. A avaliação do mAbES contou com a participação de especialistas em IHC, especialistas em videojogos (Estudo 1) e usuários finais com deficiência visual (Estudo 2). Os instrumentos utilizados na avaliação do Estudo 1 foram questionários para feedback do áudio e de avaliação de usabilidade que também inclui questões baseadas em videojogos e mecânica de jogo. Para o Estudo 2 foram aplicados questionários de avaliação de áudio e de $O \& M$ que continha questões sobre feedback tátil e facilidade de uso. Entrevistas semiestruturadas também foram aplicadas.

\section{Introdução}

Este trabalho apresenta o jogo intitulado mAbES - Mobile Audio-Based Navigation Using Virtual Environments, destinado ao desenvolvimento de habilidades de navegação (O\&M - orientação e mobilidade) por pessoas com deficiência visual. A principal questão de pesquisa foi verificar se o mAbES poderia ajudar uma pessoa que é deficiente visual a entender um espaço real, representado virtualmente sem que tal interação pudesse substituir uma visitação real. Por isso, o jogo possui desafios que devem ser resolvidos enquanto o usuário visita o museu. Desta forma, durante a navegação, o usuário final pode desenvolver habilidades de O\&M que são validadas através da construção de mapas mentais [Mioduser e Lahav 2011]. A capacidade de navegação está relacionada à capacidade da pessoa poder deslocar-se de um ponto de origem a um ponto de destino, com segurança. A navegação baseada na visão é um processo perceptivo. Mas na pessoa que é cega, a navegação passa a ser um processo mais cognitivo e requer um estado consciente de atenção a todo momento para solução de problemas [Giudice e Legge 2008]. Uma pessoa com deficiência visual deve ter 
noções de orientação e mobilidade, a fim de alcançar um bom nível de navegação, com segurança, eficiência e agilidade [Hill e Ponder 1981]. A aprendizagem de habilidades de O\&M inclui um conjunto de técnicas definidas para que as crianças, jovens e adultos (ou aqueles com deficiência visual) possam pratica-la etapa por etapa. No entanto, aprender essas habilidades também envolve outros aspectos, tais como treinamento e refinamento da percepção, e desenvolvimento de competências tanto conceituais quanto motoras [Mioduser e Lahav 2011; Hill e Ponder 1981]. O suporte para o nível perceptivo e conceitual é importante para o desenvolvimento das capacidades de navegação e para a construção de mapas mentais [Sánchez at al 2009; Mioduser e Lahav 2011; Lahav e Mioduser 2008]. Assim, o mapa mental trata de uma representação internalizada do espaço, utilizando conhecimento objetivo e percepção subjetiva.

Considerando que a vida real pode ser representada através de ambiente virtual, é possível criar aplicativos de treinamento que permitem que um usuário que é cego interaja com os elementos do ambiente simulado durante a navegação [Sánchez e Maureira 2007; Sánchez e Oyarzún 2011]. Há diferentes recursos tecnológicos que têm sido desenvolvidos para auxiliar a navegação, e, assim, permitir que os usuários cegos entendam melhor o mundo em torno deles. Há bengalas de vibração, modelos tácteis, aplicações baseadas em GPS, simuladores de ambiente interno, RFID (identificação por radiofrequência), e outros [Giudice e Legge 2008]. Algumas tecnologias apresentam foco na navegação em grande escala, em ambientes desconhecidos, ou espaços conhecidos, bem como podem ser úteis para pessoas com baixa visão. De toda a forma, para que possam trazer facilidades à vida de seus usuários, é preciso que sejam de fácil uso. Existe um esforço de pesquisadores para construir sistemas interativos que possam ser utilizados com autonomia por pessoas com deficiência visual [Sánchez et al 2009, Miodusre e Lahav 2011; Giudice e Legge 2008; Lahav e Mioduser 2008; Connors et al 2014; Yuan et al 2011; Stephanidis 2001; Sánchez et al 2014], e que sejam fáceis e simples de serem utilizados. Os termos usabilidade e acessibilidade estão relacionados e devem ser considerados ao longo das etapas de concepção, desenvolvimento e avaliação de aplicações computacionais [Stephanidis 2001]. Existem diferentes categorias de métodos de avaliação de usabilidade e de acessibilidade [Petrie e Bevan 2009]:

- Verificação automatizada de conformidade com as diretrizes e normas.

- Avaliações conduzidas por especialistas.

- Avaliação utilizando modelos e simulações.

- Avaliação com usuários ou potenciais usuários.

- Avaliação dos dados coletados durante a utilização do sistema.

A avaliação do mAbES foi conduzida com especialistas em Interação HumanoComputador e especialistas em videojogos (Estudo 1) e usuários finais ou potenciais (Estudo 2). O objetivo da avaliação foi verificar a adequação do mAbES aos critérios de usabilidade e acessibilidade e verificar se o mAbES poderia ajudar pessoas com deficiência visual a compreender um espaço que é representado por meio virtual.

\section{2. mAbES}

O mAbES - Mobile Audio-Based Navigation Using Virtual Environments - é um jogo que teve como inspiração o AbES [Sánchez et al 2009, Sánchez et al 2010], que é um videogame para plataforma desktop direcionado ao desenvolvimento de habilidades de orientação e mobilidade para usuários que são cegos. O mAbES foi desenvolvido para 
plataforma móvel e simula experimentos do Museu de Ciências e Tecnologia da Pontifícia Universidade Católica do Rio Grande do Sul. Foi concebido como um jogo inclusivo, permitindo uso tanto por usuários que são cegos quanto por usuários videntes.

A concepção do mAbES foi realizada por uma equipe interdisciplinar que incluiu a equipe da Coordenação Pedagógica do MCT. Assim, foi definido o tema do jogo: Física - Energia. Foram mapeados três experimentos: Usina Nuclear (Figura 1), Trem da Energia e Casa Genial, localizados no $3^{\circ}$ andar do museu.

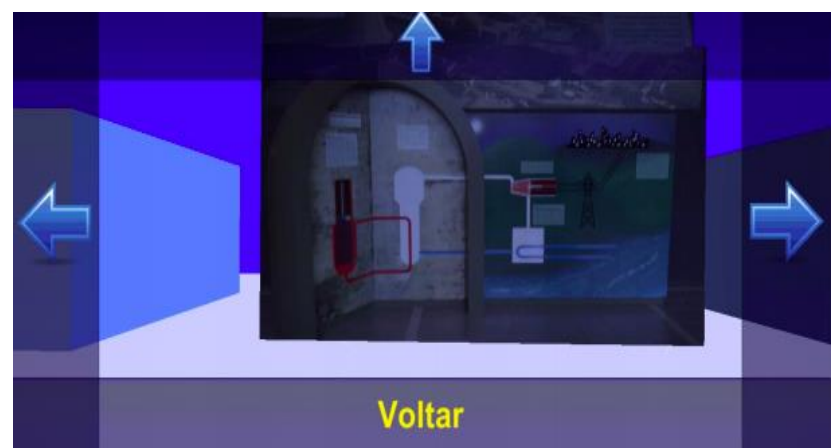

Figura 1. mAbES: Usina Nuclear

\subsection{Interface}

O mAbES possui uma interface multimodal. A interface sonora é responsável por transmitir as informações relacionadas ao ambiente do museu e aos experimentos. Desta forma, possui sons icônicos e audiodescrições, que explicam os experimentos que estão modelados no aplicativo. Os desafios que os usuários devem responder ao longo da interação com o mAbES também são apresentados. Os sons icônicos são utilizados para indicar colisões do usuário com algum objeto do museu. As informações fornecidas em áudio permitem ao usuário: 1 - Escutar, 2 - Pausar, 3 - Aumentar a velocidade, 4 Voltar, 5 - Continuar, 6 - Acessar ajuda.

A interface háptica consiste na vibração, fornecendo feedback pelo smartphone quando ocorre eventos do tipo colisão. E a interface gráfica representa em 3D os espaços do museu: tamanho, formato e posição dos espaços, dos experimentos selecionados e dos objetos (escada rolante, portas, paredes, cadeiras, mesas, estantes, etc.). Essa representação gráfica permite que o mAbES também possa ser utilizado por videntes e por pessoas com baixa visão.

\subsection{Interação}

O mAbES funciona baseado em uma metáfora de matriz Braille [Silva et al 2014], que consiste em uma estrutura matricial, de área retangular com duas colunas e três linhas (Figura 2).
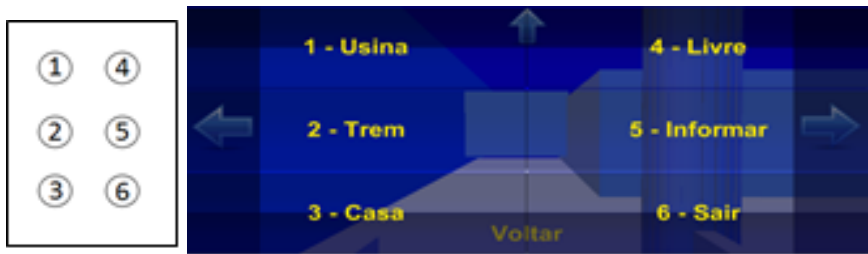

Figura 2. mAbES: Matriz Braille 
Além da matriz Braille, o mAbES utiliza o limite da tela do smartphone para a navegação, permitindo que o usuário se desloque para frente, e gire para a direita ou para a esquerda (Figura 3 ).
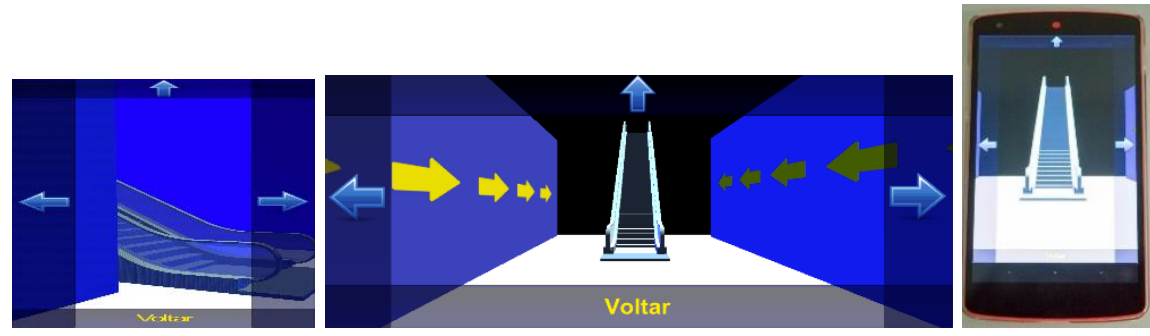

Figura 3. mAbES: Telas de navegação

\section{Avaliação de uso do mAbES}

Para avaliar o mAbES foram conduzidos dois estudos em separado. O Estudo 1 foi conduzido com especialistas em IHC e com especialistas em jogos digitais para pessoas que são cegas. $\mathrm{O}$ Estudo 2 foi conduzido com usuários finais e potenciais. Cada estudo foi conduzido em 3 etapas. A primeira se referiu a avaliação dos sons, tendo como fonte a Nota Técnica $n^{\mathbf{o}}$ 21/2012/MEC/SECADI/DPEE [Brasil 2012]. A segunda etapa se referiu a avaliação de usabilidade e de satisfação de uso, enquanto a terceira avaliou questões de orientação e mobilidade.

\subsection{Amostra}

- Estudo 1: foi composta por uma amostra intencional, e contou com a participação de três especialistas IHC e dois especialistas em audiogames e em jogos inclusivos.

- Estudo 2: após a divulgação da pesquisa em uma turma de uma escola inclusiva, foram selecionados seis alunos, que ainda não conheciam o MCT. Desses, $50 \%$ era de cada gênero. Dois participantes tinham entre 10 e 14 anos, outros dois entre 20 e 36 anos de idade e dois outros com 44 anos de idade. Dessa amostra, cinco alunos eram totalmente cegos e um possuía alguma visão residual. Os participantes foram organizados em subgrupos: o subgrupo (a) visitou o MCT utilizando o mAbES, sem ter previamente utilizado o jogo. Enquanto o subgrupo (b) utilizou o mAbES antes e durante a visitação ao museu.

\subsection{Instrumentos de avaliação}

Para a avaliação de uso do mAbES foram desenvolvidos 3 instrumentos: Instrumento de avaliação dos sons, de avaliação de usabilidade, e de avaliação de orientação e mobilidade. Esses instrumentos utilizaram a escala de Likert de 5 pontos (Concordo totalmente, Concordo parcialmente, Neutro, Discordo parcialmente e Discordo totalmente).

\subsubsection{Instrumento de avaliação dos sons}

A avaliação das informações sonoras ocorreu em duas etapas: primeiramente com os autores deste trabalho e, após, com os participantes do Estudo 1 e do Estudo 2. 
Os autores do trabalho avaliaram os sons do mAbES a partir de requisitos da Nota Técnica $n^{0}$ 21/2012/MEC/SECADI/DPEE [Brasil 2012]. No caso de não atendimento à norma, foi indicado o requisito que não estava sendo atendido e foi elaborada uma sugestão de transcrição de som. Dos 156 sons do mAbES, foram identificados 30 que deveriam ser alterados. Foi feita uma seleção descartando aqueles que se referissem ao mesmo requisito da Nota Técnica que não estava sendo atendido e ao final foram selecionados 16 sons, que foram incluídos no instrumento de avaliação para os participantes do Estudo 1 e 2.

O instrumento de avaliação de som continha a identificação dos arquivos de áudio dos 16 sons, a quantidade de vezes que o som era executado pelo usuário, a indicação de preferência pelo som original ou som sugerido, e um campo de preenchimento opcional para incluir comentários sobre cada som.

\subsubsection{Instrumento de avaliação de usabilidade e de acessibilidade}

- Estudo 1: com 35 questões sendo 28 questões baseadas nas 10 Heurísticas de Nielsen [Nielsen 1993] e em mecânica de jogos [Giudice e Legge 2008; Connors et al 2014; Yuan et al 2011; Sánchez et al 2009; Sánchez et al, 2014], 4 questões relacionados à interface háptica; e 3 questões relacionadas à interface sonora.

- Estudo 2: com 22 questões fechadas sendo 12 questões relacionadas à usabilidade, 3 relacionadas à interface sonora, 4 relacionadas à interface háptica, 2 relacionadas à interface gráfica, 1 relacionada ao Braille. Possuía, ainda, mais 5 questões abertas relacionadas à facilidade de uso e satisfação do usuário, que serviram para confirmar as respostas anteriores. Também foram realizadas entrevistas semiestruturadas.

As questões de interface sonora incluíram avaliação de sons do tipo informacional: fala, alarmes, dados sonificados. Foi avaliada a qualidade do som no que se refere à compreensão da mensagem e velocidade de execução. A avaliação da interface háptica esteve relacionada à percepção e utilização da vibração.

\subsubsection{Mecanismo de avaliação de orientação e mobilidade (O\&M)}

Após o uso do mAbES, os participantes do Estudo 1 e do Estudo 2 desenharam o ambiente do museu. Os participantes do Estudo 2 desenharam o museu em uma folha de papel sob uma folha de espuma sintética, que foi utilizada para marcar o traço e permitir o reconhecimento do desenho por meio do tato.

\subsection{Procedimentos}

A seguir são descritos os procedimentos adotados para a avaliação de uso do mAbES

\subsubsection{Avaliação da interface sonora}

Os participantes do Estudo 1 e do Estudo 2 escutaram os sons originais e os sons sugeridos quantas vezes foram necessárias para compreender os sons e para comparálos. Durante a execução, foram preenchendo o questionário de avaliação dos sons. Os questionários dos participantes do Estudo 2 foram preenchidos pelas avaliadoras. 


\subsubsection{Avaliação de usabilidade e de acessibilidade. Avaliação de orientação e mobilidade}

Aos participantes do Estudo 1 e do Estudo 2 foi explicado o que é o mAbES e o contexto em que está inserido. Após, o aplicativo pode ser utilizado sem restrição de tempo.

- Estudo 1: após utilizar livremente o mAbES, os participantes tiveram que representar graficamente o ambiente do Museu e responder ao instrumento de avaliação de usabilidade e de acessibilidade.

- Estudo 2: foi organizado em 2 subgrupos sendo que todos os participantes utilizam o mAbES durante a visitação ao museu. O subgrupo (a) não utilizou o mAbES previamente enquanto o subgrupo (b) utilizou o mAbES antes da visitação ao museu. Todos os participantes receberam a seguinte tarefa: Você está no andar térreo do Museu, próximo à entrada. Deverá ir ao terceiro andar, e explorar o experimento da Usina Nuclear. Após, deverá explorar a Casa Genial. Na Usina Nuclear deverá escutar as informações e responder aos desafios. Na Casa Genial deverá entrar e observar o que há na sala. O Subgrupo (a) respondeu o instrumento de avaliação de usabilidade e de acessibilidade e desenhou os espaços do museu após a visitação. O subgrupo (b) respondeu ao questionário de usabilidade e representou graficamente o ambiente do museu, a partir da interação com o aplicativo, e antes da visitação. Depois da navegação in loco, os usuários tiveram que confirmar as respostas já fornecidas e redesenhar o ambiente. Os usuários do Estudo 2, que utilizaram fones de ouvido durante a visitação, relataram em voz alta o que acontecia enquanto navegavam com o mAbES.

\section{Resultados}

\subsection{Avaliação da interface sonora}

- Estudo 1: somente um especialista em IHC preferiu um conjunto de 5 sons originais ao invés das respectivas sugestões. Os demais especialistas em IHC preferiram os sons sugeridos. Todos os especialistas em jogos preferiram os sons sugeridos.

- Estudo 2: os participantes escolheram pelo menos um som original ao invés do som sugerido. A relação de preferência pelos sons sugeridos em relação aos sons originais foi de $71 \%$ para $29 \%$. Segundo os participantes, por vezes o atendimento à Norma Técnica [Brasil 2012] fez com que alguns sons tivessem informações excessivas, tornando-os mais extensos e cansativos.

\subsection{Avaliação de usabilidade e de acessibilidade}

Para o trabalho aqui apresentado, as categorias "Concordo plenamente" e "Concordo parcialmente" foram agrupadas em "Concordo" e as categorias "Discordo totalmente" e "Discordo parcialmente" foram agrupadas em "Discordo". Esse agrupamento foi possível porque não traz impactos significativos nos resultados. 
- Estudo 1: o instrumento foi organizado considerando as heurísticas de Nielsen [Nielsen 1993], a interface sonora e a interface háptica (Tabela 1).

Tabela 1. Estudo 1: Resultado quantitativo

\begin{tabular}{|c|c|c|c|}
\hline \multicolumn{1}{|c|}{ Categoria } & Questões & Concordo & Discordo \\
\hline $\begin{array}{l}\text { 10 Heurísticas de Usabilidade para } \\
\text { Interfaces de usuários }\end{array}$ & $\mathbf{2 8}$ & $\mathbf{6 3 , 5 \%}$ & $\mathbf{3 6 , 5 \%}$ \\
\hline -Visibilidade do estado do sistema & 3 & $53,3 \%$ & $46,7 \%$ \\
\hline -Relação entre sistema e mundo real & 4 & $85 \%$ & $15 \%$ \\
\hline -Controle e liberdade do usuário & 3 & $26,6 \%$ & $73,3 \%$ \\
\hline $\begin{array}{c}\text {-Consistência e padronização } \\
\text { (guidelines) }\end{array}$ & 2 & $90 \%$ & $10 \%$ \\
\hline -Prevenção de erros & 2 & $20 \%$ & $80 \%$ \\
\hline $\begin{array}{c}\text {-Reconhecimento ao invés de } \\
\text { memorização }\end{array}$ & 3 & $86,7 \%$ & $13,3 \%$ \\
\hline -Flexibilidade e eficiência de uso & 3 & $66,7 \%$ & $33,3 \%$ \\
\hline -Projeto estético e minimalista & 2 & $80 \%$ & $20 \%$ \\
\hline $\begin{array}{c}\text {-Reconhecimento, diagnóstico e } \\
\text { recuperação de erros }\end{array}$ & 3 & $60 \%$ & $40 \%$ \\
\hline -Ajuda e documentação & 3 & $66,7 \%$ & $33,3 \%$ \\
\hline Interface Sonora & $\mathbf{3}$ & $\mathbf{8 6 , 7 \%}$ & $\mathbf{1 3 , 3 \%}$ \\
\hline \begin{tabular}{c} 
Interface háptica \\
\hline
\end{tabular} & $\mathbf{1 0 0 \%}$ & $\mathbf{0 \%}$ \\
\hline
\end{tabular}

- Estudo 2: o subgrupo (a) concordou com 88,2\%, discordou com 8,5\% e se mostrou indiferente com 3,3\% das questões. No que se refere ao subgrupo (b), as diferenças significativas estiveram relacionadas às questões inicialmente sinalizadas como indiferentes. Pré-teste: $80,3 \%$ concordam, $10,8 \%$ discordam e $8,9 \%$ indicaram indiferença. Pós-teste: $82,5 \%$ concordam, $10,5 \%$ discordam e $7 \%$ indicaram indiferença. As respostas às questões abertas junto à observação de uso do mAbES e a visitação ao museu permitiu fazer algumas observações:

o Rotação: os giros são feitos baseados nas horas do relógio. Desta forma, um giro de $90^{\circ}$ requer 3 cliques no botão direito. Esta relação poderia ser mais destacada.

○ Interface gráfica: as informações poderiam ser ajustáveis e personalizáveis para permitir o zoom, quando desejado.

o Rotas: quando o usuário sai de uma rota esperada, o aplicativo não fornece ajuda para que ele possa reconhecer o ambiente e retornar ao ponto desejado.

o Feedback háptico: poderia ser ajustável às preferências dos usuários. A pesquisa mostrou que esse recurso foi mais útil durante as primeiras interações. Com o uso do mAbES, os usuários se detiveram mais às informações sonoras. 
- Sistema de ajuda: deveria ser contextual para ajudar o usuário a se localizar no museu bem como conforme os desafios que precisa responder.

o Posição do usuário: o mAbES necessita de um recurso que indique o local em que o usuário se encontra no espaço virtual do museu, bem como poderia fornecer informações para que pudesse reconhecer o espaço a sua volta. Esta foi uma questão recorrente.

\subsection{Avaliação de orientação e mobilidade}

- Estudo 1: conseguiram compreender o espaço do museu a partir do uso do mAbES e o representaram de diferentes formas (Figura 4). Destaca-se que esses participantes são videntes e utilizaram o mAbES sem restrições.

- Estudo 2: a maioria dos participantes não tinha experiência em fazer desenhos (Figura 5 - Subgrupo (a), Figura 6 - Subgrupo (b)). Participantes do subgrupo (b) puderam refinar a compreensão do espaço ao utilizar o mAbES antes de fazer a visita ao museu.
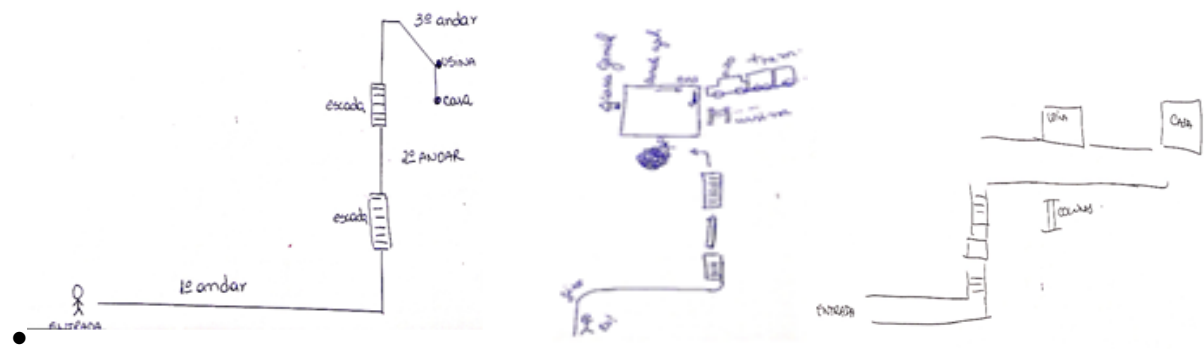

Figura 4. Representação do espaço do museu pelos autores (a). Estudo 1: Exemplos de representações do museu (b)(c)
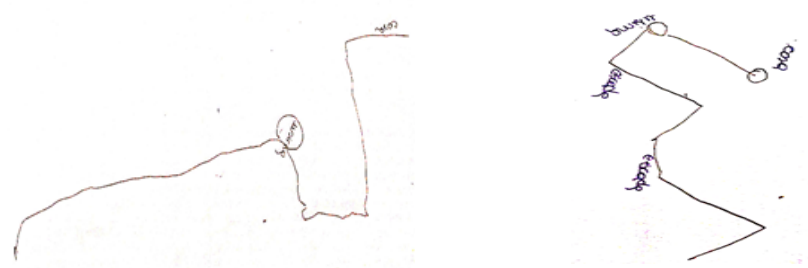

Figura 5. Estudo 2 - Subgrupo (a): Exemplos de representações do museu

Figura 6. Estudo 2 - Subgrupo (b): desenho em pré-teste e pós-teste

\section{Considerações finais}

Este artigo trata sobre a avaliação de usabilidade de um aplicativo móvel de navegação para pessoas cegas, o mAbES. A avaliação do mAbES foi realizada com especialistas 
em IHC e especialistas em videojogos (Estudo 1) e usuários ou potenciais usuários (Estudo 2). O Estudo 1 indicou que o mAbES responde à maioria dos critérios de usabilidade definidos por Nielsen [Nielsen 1993] e bem como aprovaram as sugestões de sons.

O Estudo 2 teve participantes que nunca tinham desenhado e, mesmo assim, conseguiram estabelecer relações espaciais entre os experimentos e o espaço que eles ocupam no museu. $\mathrm{O}$ uso prévio do mAbES a visita permitiu que os usuários pudessem explorar o museu com maior autonomia e segurança durante a visitação.

No que se refere ao projeto e desenvolvimento de aplicativos similares, destacam-se algumas sugestões e cuidados que devem ser considerados para que usuários que são cegos possam melhor utilizar um sistema baseado em interface sonora, háptica e gráfica. São eles:

- Uso de audiodescrição para descrever as imagens e os espaços que são representados na aplicação.

- Liberdade de uso do usuário para controlar a execução das informações sonoras e hápticas.

- Inserção de um sistema de ajuda contextual, que possa auxiliar o usuário a reconhecer o espaço em que se encontra bem como as atividades que deve realizar.

- Prevenção de erro que ode ser acionada quando o usuário executa uma ação não esperada pelo software.

- Priorizar as informações sonoras ao invés de detalhar a interface gráfica.

- Manter uma padronização nas ações esperadas pelo usuário no que se refere à interação com o software.

\section{Agradecimentos}

Este trabalho teve o apoio do Programa STIC AmSud-CAPES/CONICYT/MAEE, Projeto KIGB-Knowing and Interacting while Gaming for the Blind, 2014, do Museu de Ciência e Tecnologia, da PUCRS e do Prof. Dr. Jaime Sánchez, que participou de discussões para a realização do Trabalho de Conclusão de Curso, que forneceu dados para o trabalho aqui apresentado.

\section{Referências}

BRASIL. Ministério Da Educação. Nota Técnica nº 21 / 2012 / MEC / SECADI /DPEE. Orientações para descrição de imagem na geração de material digital acessível Mecdaisy (2012)

Connors, E. C., Yazzolino, L. A., Sánchez, J., Merabet, L. B. Development of a Audiobased Virtual Gaming Environment to Assist with Navigation Skills in the Blind. J Vis Exp. Author manuscript; available in PMC 2014. http://www.ncbi.nlm.nih.gov/pmc/articles/PMC3641639/pdf/nihms546296.pdf (2014) 
Giudice, N. A., Legge, G. E. Blind navigation and the role of technology. In: A. Helal, M. Mokhtari \& B. Abdulrazak (Eds.), Engineering handbook of smart technology for aging, disability, and independence (pp. 479-500): John Wiley \& Sons. (2008)

Hill, E., Ponder, P. Orientación y técnicas de Movilidad, Una guía para el practicante. México: Comité internacional pro-ciegos. (1981)

Lahav, O., Mioduser, D. Haptic-feedback support for cognitive mapping of unknown spac-es by people who are blind, International Journal of Human-Computer Studies, 66(1), pp. 23-35. (2008)

Mioduser, D., Lahav, O. Blind persons' acquisition of spatial cognitive mapping and orientation skills supported by virtual environment. International Journal on Disability and Human Development. 4(3), 231-238. Print (2005). Online. (2011)

Nielsen, J. Usability Engineering. California: Elsevier, 362p (1993)

Petrie H., Bevan, N. The evaluation of accessibility, usability and user experience. In: The Universal Access Handbook, C Stepanidis (ed), CRC Press (2009)

Sánchez, J., Campos, M. B., Espinoza, M. Multimodal Gaming for Navigation Skills in Players Who Are Blind. In: XIII Brazilian Symposium on Human Factors in Computer Systems. SBC (2014)

Sánchez, J., Maureira, E. Subway Mobility Assistance Tools for Blind Users. Universal Access in Ambient Intelligence Environments, Lecture Notes in Computer Science, Volume 4397, pp 386-404. (2007).

Sánchez, J., Oyarzún, C. Mobile Audio Assistance in Bus Transportation for the Blind. In: International Journal on Disability and Human Development (IJDHD) 10(4), pp. 365-371. (2011)

Sánchez, J., Sáenz, M., Pascual-Leone, A., Merabet, L. Enhancing navigation skills through audio gaming. Proc. CHI EA '10, ACM, New York, NY, USA, pp. 39913996 (2010)

Sánchez, J., Tadres, A., Pascual-Leone, A., Merabet, L. Blind Children Navigation through Gaming and Associated Brain Plasticity, In Proc. of the Virtual Rehabilitation 2009 International Conference, Haifa, Israel, IEEE, pp. 29-36. (2009)

Silva, A. R. S.; Façanha, A. R.; Viana, W.; Castro Filho, J. A..; Sánchez, J. Especificação e desenvolvimento de um ambiente educativo móvel para a prática da escrita Braille. In: Simpósio Brasileiro de Informática na Educação, SBIE, 2014. http://www.br-ie.org/pub/index.php/sbie/article/view/2971 (2014)

Stephanidis, C. User Interfaces for All: New perspectives into Human-Computer Interaction. In: C. Stephanidis (Ed.), User Interfaces for All - Concepts, Methods, and Tools. pp. 3-17. Mahwah, NJ: Lawrence Erlbaum Associates (2001).

Yuan, B., Folmer, E., Harris, F. C. Jr. Game accessibility: a survey. In: Universal Access in the Information Society. 10(1), pp. 81-100. Springer-Verlag Berlin, Heidelberg (2011) 